\title{
Nonlinear Vibration Analysis of Flexible Hoisting Rope with Time-Varying Length
}

\author{
Ji-hu Bao \\ Hefei General Machinery Research Institute, Hefei 230031, China \\ School of Mechanical Engineering, Shanghai Jiaotong University, Shanghai 200240, China \\ Peng Zhang, Chang-ming Zhu and Ming Zhu \\ School of Mechanical Engineering, Shanghai Jiaotong University, Shanghai 200240, China
}

(Received 3 March 2013; accepted 14 January 2015)

The nonlinear vibration of a flexible hoisting rope with time-varying length and axial velocity is investigated. The flexible hoisting rope is modeled as a taut translating string with a rigid body attached at its low end. A systematic procedure for deriving the system model of a flexible hoisting rope with time-varying length and axial velocity is presented. The governing equations were developed by employing the extended Hamilton's principle considering coupling of axial movement and flexural deformation of the rope. The derived governing equations are nonlinear partial differential equations(PDEs) with time-varying coefficients. The Galerkin's method and the $4^{\text {th }}$ RungeKutta method were employed to numerically analyze the resulting equations. Further, the dynamic stability of the flexible hoisting rope was investigated according to the Lyapunov stability theory. The motions of an elevator hoisting system were presented to illustrate the proposed mathematical models. The results of simulation show that the dynamic motions of the flexible hoisting string are stable during downward movement but are unstable during upward movement. The proposed systematic procedures in analyzing the dynamic stability can facilitate further development in dynamic control of the flexible hoisting system in practice.

\section{NOMENCLATURE}

\section{$a$}

\section{A, B}

C

$d$

$E$

$E_{k}$

$E_{e}$

$E_{g}$

$g$

Damp matrixes system (J)

Integer

Inertia $\left(\mathrm{m}^{4}\right)$

Integer

Integer

Mass matrixes

Time (s)
Axial acceleration of the string $\left(\mathrm{m} / \mathrm{s}^{2}\right)$

Matrix differential operators

Diameter of the string (m)

Young's Modulus of the string $(\mathrm{Pa})$

Kinetic energy of flexible hoisting system $(\mathrm{J})$

Elastic strain energy of the string $(\mathrm{J})$

Gravitational potential energy of flexible hoisting

Gravitational constant $\left(\mathrm{m} / \mathrm{s}^{2}\right)$

Unit vector along the $\mathrm{x}$-axes

$n \times n$ identity matrix

Unit vector along the $y$-axes

Stiffness matrixes

Length of the string (m)

Mass of rigid body $(\mathrm{kg})$

Number of included modes

Longitudinal tension (N)

Generalized coordinates

Vectors of generalized coordinates

Position vector of the string

Position vector of the rigid body

Lyapunov candidate function
$U \quad$ State vector

$v \quad$ Axial velocity of the string $(\mathrm{m} / \mathrm{s})$

V Velocity vector of the string

$\mathbf{V}_{\mathbf{c}}$ Velocity vector of the rigid body

$x \quad$ Spatial variable $(\mathrm{m})$

$y \quad$ Transverse displacement of the string (m)

$\zeta \quad$ Transformed spatial variable

$\varepsilon \quad$ Strain measure

$\rho \quad$ Linear density of the string $(\mathrm{kg} / \mathrm{m})$

$\lambda_{k} \quad$ Eigenvalue $(k=1,2,3,4)$

$\xi_{k} \quad$ Real parts of eigenvalue $(k=1,2,3,4)$

$\varphi_{i} \quad$ Trial function used in Eq. (19)

$\delta_{i j} \quad$ Kronecker delta

$\omega_{k} \quad$ Imaginary parts of eigenvalue $(k=1,2,3,4)$

$\Lambda \quad$ Eigenvector

\section{INTRODUCTION}

Ropes with time-varying length are widely used in the hoisting industry such as mine hoists, elevators, cranes, etc; They are subject to vibration due to their high flexibility and relatively low internal damping characteristics., ${ }^{1,2}$ Most often these systems are modeled as either an axially moving tensioned beam or as a string with time-varying length and a rigid body at its lower end. ${ }^{3,4}$ It was reported that the vibration energy of the rope changed in general during elongation and shortening. ${ }^{5-7}$ Zhang $^{8-11}$ and Bao ${ }^{12,13}$ published a series of studies on vibration of a flexible hoisting system with arbitrarily varying length. Terumichi et al. assumed the velocity of the string was constant and studied the transverse vibrations of a string with time-varying length and a mass-spring system 